\title{
Serum Heparanase: A New Clinical Biomarker Involved in Senile Metabolic Inflammatory Syndrome
}

\author{
Qingsong Wang' \\ Xiu-ling Zhou ${ }^{2}$ \\ Guang-hai $\mathrm{Mei}^{3}$ \\ Jiantao Wang' \\ Zhongyu $\mathrm{Yu}^{\prime}$ \\ Yue Hu' \\ Xinyu Guo' \\ Hanyu Zhang' \\ Xiaodie $\mathrm{Xu}^{4}$ \\ Chengfeng $\mathrm{He}^{4}$ \\ Jingchun Guo \\ Yinghong Tao ${ }^{5}$ \\ Houguang Zhou'
}

'Department of Geriatrics, Huashan Hospital, National Clinical Research Center for Aging and Medicine, Fudan University, Shanghai, 200040, People's Republic of China;

${ }^{2}$ Department of Ultrasonics, Huashan Hospital, Fudan University, Shanghai, 200040, People's

Republic of China; ${ }^{3}$ Department of

Neurosurgery, Huashan Hospital, Fudan

University, Shanghai, 200040, People's Republic of China; ${ }^{4}$ National Key Laboratory of Medical Neurobiology, Institute of Brain Science, Fudan University, Shanghai, 200030, People's Republic of China; ${ }^{5}$ Department of Medical Examination Center, Huashan Hospital, Fudan University, Shanghai, 200040, People's Republic of China

Correspondence: Houguang Zhou

Department of Geriatrics of Huashan Hospital, National Clinical Research Center for Aging and Medicine, Fudan University, No. 24 Ward, Building 6, No. 12 Middle WuLuMuQi Road, JingAn District, Shanghai, 200040, People's

Republic of China

Tel +86-2I-52887246

Email zhouhouguang@huashan.org.cn

Yinghong Tao

Department of Medical Examination Center of Huashan Hospital, Fudan University, Building 4,

No. 12 Middle WuLuMuQi Road, JingAn

District, Shanghai, 200040, People's Republic of

China

Tel +86-21-52889087

Email taoyinghong@huashan.org.cn
Aim: Metabolic inflammation syndrome (MIS) can lead to a series of complications, but its exact inflammatory mechanism is still unclear. The aim of this study was to explore the correlation between heparanase (HPA) and MIS, and the close relationship between HPA and other chronic low-grade inflammation index, such as C-reactive protein (CRP) and interleukin-6 (IL-6).

Methods: A total of 105 patients with MIS in the physical examination population of Huashan Hospital affiliated to Fudan University from May to June 2018 were selected as the MIS group, and 52 patients who were relatively healthy during the same period were used as the control group. The basic clinical data of the selected candidates were collected, the levels of serum HPA, CRP and IL-6 were measured by ELISA, and the levels of blood glucose and blood lipids were also detected.

Results: Compared with the control group, the levels of HPA, CRP, IL-6, FBG, HbA $1 \mathrm{C}$, and TG of MIS group were all significantly elevated (all $\mathrm{P}<0.05$ ), and HDL-C levels were considerably reduced $(\mathrm{P}<0.05)$. Correlation analysis showed that there was a noticeably positive correlation between serum HPA level and CRP, IL-6 levels $(\mathrm{P}<0.05)$.

Conclusion: Higher HPA levels might play a certain role in the occurrence and development of MIS. There was a certain close correlation between serum HPA level and CRP and IL-6 levels, and which indicated that HPA was involved in the chronic low-grade inflammatory reaction process of MIS.

Keywords: metabolic inflammatory syndrome, MIS, heparanase, HPA, C-reactive protein, CRP, interleukin-6, IL-6, chronic low-grade inflammation, CLGI

\section{Introduction}

Metabolic Inflammatory Syndrome (MIS) is a novel concept of holistic integrative medicine for management of metabolic diseases, which encompasses atherosclerosis (AS), type 2 diabetes mellitus (T2DM), non-alcoholic fatty liver disease (NAFLD), and obesity. MIS is diagnosed in patients with two or more of the above four metabolic conditions. ${ }^{1}$ Studies have shown that in patients with T2DM, the detection rate of MIS is higher than that of metabolic syndrome (MS), so MIS is more likely to occur in inflammation-related metabolic diseases. ${ }^{1}$ The concept and diagnosis of MIS will help the early detection of AS, T2DM, NAFLD and obesity, and will encourage early screening for AS in patients with T2DM, NAFLD and obesity.

Heparanase (HPA) is associated with inflammatory responses, and various immune cells including macrophages could secrete HPA. ${ }^{2}$ The inflammatory 
effect of HPA is closely related to its degradation of heparan sulfate proteoglycan (HSPG). The islet basement membrane is rich in heparan sulfate proteoglycans, which protects the islets from immune destruction. The destruction of this structure is an indication of the occurrence of autoimmune pancreatitis or diabetes. ${ }^{3}$ The study of Shafat et $\mathrm{al}^{4}$ found that serum HPA levels in patients with T2DM were significantly higher than in normal people. Therefore, HPA may play an important role in the occurrence and development of T2DM. Related studies have shown that the level and activity of HPA in atherosclerotic lesions increases as the disease progresses, and that the level and activity of HPA in unstable plaques are significantly higher than those in stable plaques, indicating that HPA is not only involved in the formation of initial plaque lesions, but also in the evolution of stable plaques into vulnerable plaques. ${ }^{5}$ Muhammad et $\mathrm{al}^{6}$ used ApoE-deficient mice and found that hepatic steatosis was reduced in mice injected with the HPA inhibitor PG545, and the weight gain was reduced compared with mice not injected with PG545, indicating that HPA and liver steatosis related to weight gain. Therefore, previous research suggests that there is a certain correlation between the four components of MIS and HPA. However, there is no report on the correlation between HPA and MIS. Therefore, this article intends to explore the intrinsic correlation between HPA and MIS by detecting serum HPA levels and chronic low-grade inflammation (CLGI) related indicators CRP, IL-6 levels in patients with MIS.

\section{Materials and Methods}

\section{Participants and Ethics}

One hundred and five cases of MIS patients in the geriatric physical examination population of Huashan Hospital affiliated to Fudan University from May to June 2018 were selected as the case group, aged 6085 years; 52 cases of relatively healthy physical examination during the same period were selected as the control group, aged 60-85 years. All subjects signed the informed consent. This study was performed in accordance with the Declaration of Helsinki (2013) of the World Medical Association, and approved by the ethics committee of Huashan Hospital, Fudan University (No.2015-127).

\section{Inclusion Criteria and Exclusion Criteria}

Patients with at least two of the following four diagnoses (AS, T2DM, NAFLD, or obesity) were diagnosed as MIS. ${ }^{1}$ According to the diagnostic criteria of MIS, patients diagnosed as MIS were included in the case group. And the diagnostic criteria of the four diagnoses were as follows:

1. The diagnosis of T2DM is based on the diagnostic criteria for diabetes established by the World Health Organization (WHO) in $1999 .^{7}$

2. The diagnosis of NAFLD was based on the "World Gastroenterology Organisation global guidelines: Nonalcoholic fatty liver disease and nonalcoholic steatohepatitis“ developed in 2014 . $^{8}$

3. The diagnosis of AS is based on the patient's related auxiliary examinations. ${ }^{9}$

4. $\mathrm{BMI} \geq 25 \mathrm{~kg} / \mathrm{m}^{2}$ was used as a diagnostic standard for obesity.

Exclusion criteria:

1. Type 1 diabetes, severe diabetic complications, other special types of diabetes,

2. History of alcohol abuse or alcohol consumption converted to ethanol is more than $140 \mathrm{~g} /$ week for men and $70 \mathrm{~g} /$ week for women,

3. Autoimmune diseases, such as rheumatoid arthritis, systemic lupus erythematosus, ankylosing spondylitis, etc,

4. Acute and chronic infections,

5. Patients with malignant tumors or after surgery for malignant tumors,

6. Severe organ dysfunction,

7. Hyperthyroidism or hypothyroidism,

8. Patients with mental illness.

\section{Clinical Data}

The participants' clinical information was collected by trained physicians through face-to-face inquiry, including: age, gender, alcohol consumption, previous medical history.

\section{Anthropometric and Blood Pressure Measurements}

All participants were measured for height $(\mathrm{cm})$ and weight $(\mathrm{kg})$. Weight was measured with a digital scale with an accuracy of 0.1 kilogram. The height was measured barefoot, and the accuracy of height meter was $1 \mathrm{~cm}$. Blood pressure was measured with an electronic sphygmomanometer, after 15 -min rest in a sitting position. 


\section{Laboratory Procedures}

All the subjects were taken fasting elbow vein blood in the resting state of the next morning after fasting for 8 hours, and immediately sent them to the laboratory for detecting. Biochemical markers were detected on the Hitachi 7600-020 automatic analyzer. Another $0.5 \mathrm{~mL}$ of serum was stored in a refrigerator at $-80^{\circ} \mathrm{C}$ for the detection of HPA, CRP and IL6. Cholesterol (CHOL), triglyceride (TG), high density lipoprotein cholesterol (HDL-C), and low density lipoprotein cholesterol (LDL-C) were analyzed using a fully automated biochemical analyzer (Model 7600 Series, Hitachi, Tokyo, Japan). Glucose oxidase method was used to measure FBG, and $\mathrm{HbA}_{1 \mathrm{C}}$ was measured by high performance liquid chromatography. ELISA was used to determine the levels of HPA, CRP, and IL-6 (Shanghai Enzyme-linked Biotechnology Co., Ltd.), according to the manufacturer's instructions. Briefly, anti-HPA, anti-CRP and anti-IL-6 monoclonal $\mathrm{Ab}$ were applied in microwells for the measurement of HPA, CRP, and IL-6 in the samples. The operation was carried out in strict accordance with the instructions, and then the absorbance was measured at $450 \mathrm{~nm}$ wave length. Finally, the sample concentration was calculated according to the standard curve. Ultrasound examinations of carotid arteries, vertebral arteries, lower extremity arteries and liver were carried out.

\section{Statistical Analyses}

SPSS 23.0 software was used for statistical analysis. Measurement data were expressed as mean \pm standard deviation. The $t$-test was used for comparison between the measurement data that met the normal distribution, and the rank sum test was used for the comparison between measurement data that did not meet the normal distribution. Qualitative variables were analyzed by the chi squared test. The correlation of each detection index was analyzed by Spearman or partial correlation analysis. $\mathrm{P}<0.05$ indicated that the difference was statistically significant.

\section{Results}

\section{General Characteristics of the MIS Group and the Control Group}

As shown in Table 1. Compared with the control group, the MIS group showed significantly higher BMI and the prevalence of essential hypertension $(\mathrm{P}<0.05)$, but there was no significant difference between the two groups in terms of sex, age, serum creatinine, albuminuria and the prevalence of benign prostatic hyperplasia, hyperuricemia and atrial fibrillation $(\mathrm{P}>0.05)$. In addition, MIS patients had other burden of coronary heart disease and cerebral infarction.

\section{There Was Obvious Disorder of Glucose and Lipid Metabolism in the MIS Group}

As shown in Figure 1. Compared with the control group, the MIS group were significantly increased in FBG [6.82 \pm 1.44 vs $5.00 \pm 0.39(\mathrm{P}<0.001)], \mathrm{HbA}_{1 \mathrm{C}}[6.88 \pm 0.90$ vs 5.59 $\pm 0.25 \quad(\mathrm{P}<0.001)], \quad$ TG $\quad[1.88 \pm 1.78 \quad$ vs $\quad 1.14 \pm 0.57$ $(\mathrm{P}<0.001)]$, while the MIS group were significantly reduced in HDL-C $[1.15 \pm 0.25$ vs $1.30 \pm 0.28(\mathrm{P}<0.001)]$, CHOL [4.36 \pm 0.90 vs $4.70 \pm 0.95(\mathrm{P}=0.032)]$, LDL-C $[2.56$ \pm 0.85 vs $2.90 \pm 0.76(\mathrm{P}<0.01)]$.

The Levels of HPA, CRP and IL-6 in the MIS Group Were All Significantly Higher Than the Control Group

As shown in Figure 2, the MIS group showed significantly higher level in HPA [19.38 \pm 11.08 vs $14.18 \pm 6.90$ $(\mathrm{P}=0.012)]$, CRP $[1.34 \pm 0.77$ vs $1.00 \pm 0.51 \quad(\mathrm{P}=0.018)]$,

Table I Clinical Characteristics of the MIS and the Control Group

\begin{tabular}{|c|c|c|c|}
\hline & MIS(\%) & Control (\%) & $P$ value \\
\hline $\mathbf{N}$ & 105 & 52 & \\
\hline \multicolumn{4}{|l|}{ Sex } \\
\hline Male (\%) & $95(90.48)$ & $48(92.31)$ & 0.705 \\
\hline Female (\%) & $10(9.52)$ & $4(7.69)$ & \\
\hline Age (years) & $74.36 \pm 6.18$ & $73.7 I \pm 6.80$ & 0.587 \\
\hline BMI $\left(\mathrm{kg} / \mathrm{m}^{2}\right)$ & $25.65 \pm 2.76$ & $23.68 \pm 2.85$ & 0.000 \\
\hline $\mathrm{SBP}(\mathrm{mmHg})$ & $|45.6| \pm 17.36$ & $137.19 \pm 17.23$ & 0.003 \\
\hline $\mathrm{DBP}(\mathrm{mmHg})$ & $77.66 \pm 10.80$ & $75.92 \pm 8.67$ & 0.315 \\
\hline $\begin{array}{l}\text { Serum creatinine } \\
(\mu \mathrm{mol} / \mathrm{L})\end{array}$ & $76.19 \pm 16.72$ & $79.02 \pm 15.58$ & 0.309 \\
\hline Albuminuria (\%) & $16(15.24)$ & $3(5.77)$ & 0.146 \\
\hline \multicolumn{4}{|l|}{ Concomitant disease } \\
\hline $\begin{array}{l}\text { Essential } \\
\text { hypertension (\%) }\end{array}$ & $84(80.00)$ & $18(34.62)$ & 0.000 \\
\hline $\begin{array}{l}\text { Coronary heart } \\
\text { disease (\%) }\end{array}$ & $29(27.62)$ & NA & \\
\hline $\begin{array}{l}\text { Cerebral } \\
\text { infarction (\%) }\end{array}$ & $9(8.57)$ & NA & \\
\hline $\begin{array}{l}\text { Benign prostatic } \\
\text { hyperplasia (\%) }\end{array}$ & $68(64.76)$ & $37(71.15)$ & 0.423 \\
\hline Hyperuricemia (\%) & $30(28.57)$ & $9(|7.3|)$ & 0.124 \\
\hline Atrial fibrillation(\%) & $9(8.57)$ & $\mathrm{I}(\mathrm{I} .92)$ & 0.208 \\
\hline
\end{tabular}

Notes: data are expressed as mean $( \pm S D)$ and absolute number (percentage); $\mathrm{P}<0.05$ is considered statistically significant.

Abbreviations: BMI, body mass index; SBP, systolic blood pressure; DBP, diastolic blood pressure; NA, not applicable. 

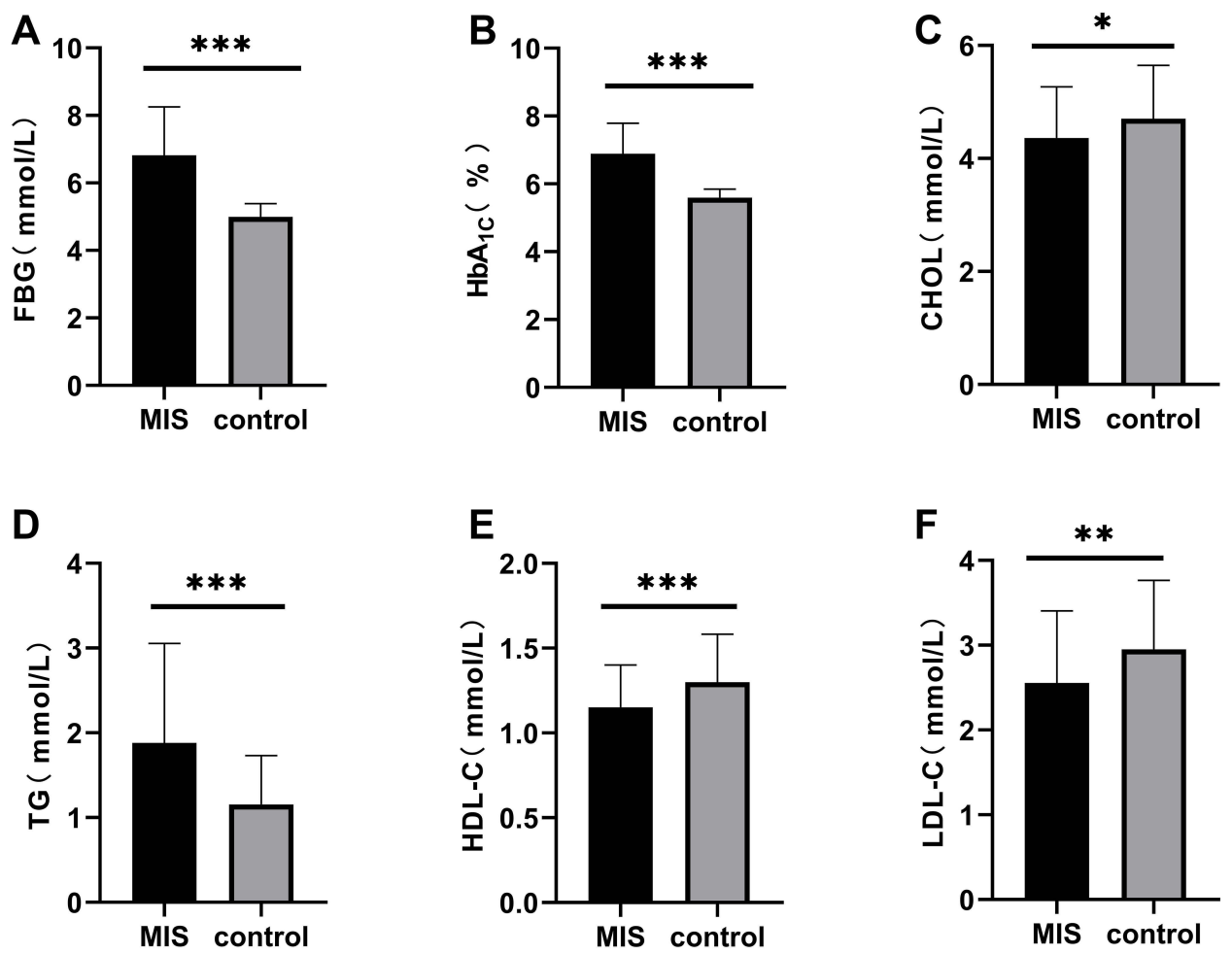

Figure I Comparison of the general biochemical characteristics of the MIS group and the control group: (A) FBG, (B) HbA IC, (C) CHOL, (D) TG, (E) HDL-C and (F) LDL$\mathrm{C}$ in MIS group and the control group; data are expressed as mean $( \pm \mathrm{SD}) ; \mathrm{P}<0.05$ is considered statistically significant; *, ** and *** indicate $\mathrm{P}$-value less than $0.05,0.0 \mathrm{I}$ and 0.001 , respectively.

Abbreviations: FBG, fasting blood glucose; $\mathrm{HbA}_{\mathrm{IC}}$, glycosylated hemoglobin; $\mathrm{CHOL}$, cholesterol; TG, triglyceride; HDL-C, high density lipoprotein cholesterol; LDL-C, low density lipoprotein cholesterol.

and IL-6[5.57 \pm 3.14 vs $4.39 \pm 2.25(\mathrm{P}=0.042)]$, compared with the control group.

\section{Correlations Between Serum HPA and} CRP, IL-6 and Clinical Indicators

As shown in Figure 3, there was a significant positive correlation between serum HPA level and CRP ( $\mathrm{r}=0.856$, $\mathrm{P}<0.001)$, IL-6 levels $(\mathrm{r}=0.832, \mathrm{P}<0.001)$, and a weakly negative between HPA and age $(r=-0.177, \mathrm{P}=0.027)$, but there was no apparent correlation between serum HPA level and FBG, $\mathrm{HbA}_{1 \mathrm{C}}, \mathrm{CHOL}, \mathrm{TG}, \mathrm{HDL}-\mathrm{C}$, and LDL-C.

\section{Discussion}

In 2016, the concept of MIS was first proposed by professor $\mathrm{Hu},{ }^{1}$ based on 4 metabolic diseases including T2DM, AS, NAFLD and obesity. The diagnosis of MIS could be made when a patient had at least 2 of these metabolic conditions. Of course, the most common constituent
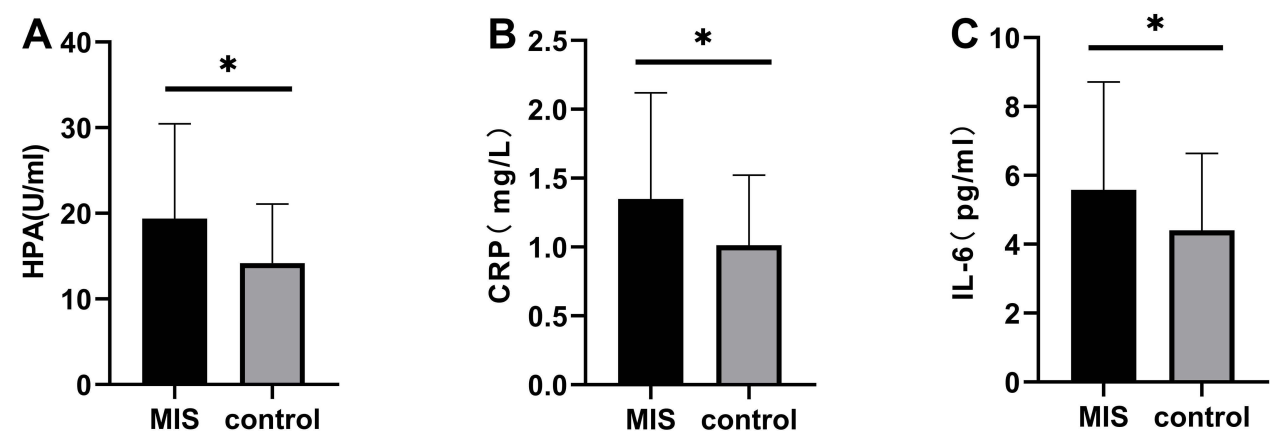

Figure 2 Comparison of the specific biochemical characteristics of the MIS group and the control group: (A) HPA, (B) CRP and (C) IL-6 in MIS group and the control group; data are expressed as mean $( \pm \mathrm{SD})$; HPA, heparanase; CRP, C-reactive protein; IL-6, Interleukin-6; P<0.05 is considered statistically significant; * indicate P-value less than 0.05 . 

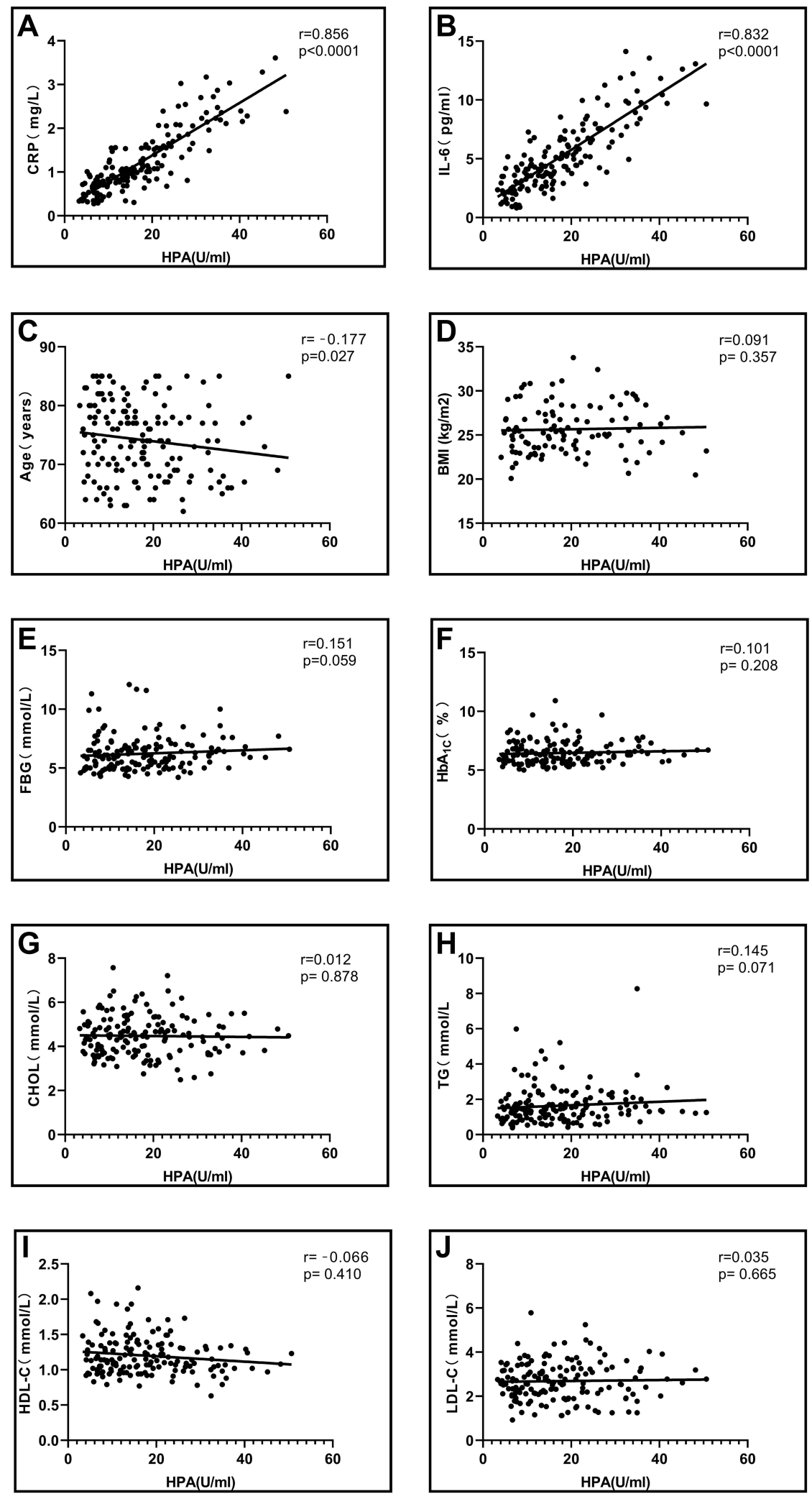

Figure 3 Correlations between serum HPA and CRP, IL-6 and clinical indicators in all subjects: Relationships between serum HPA and (A) CRP, (B) IL-6, (C) age, (D) BMI, (E) FBG, (F) HbAIC, (G) CHOL, (H) TG, (I) HDL-C, (J) LDL-C. P $<0.05$ is considered statistically significant.

Abbreviations: $\mathrm{BMI}$, body mass index; FBG, fasting blood glucose; $\mathrm{HbA}_{I C}$, glycosylated hemoglobin; $\mathrm{CHOL}$, cholesterol; TG, triglyceride; HDL-C, high density lipoprotein cholesterol; LDL-C, low density lipoprotein cholesterol; HPA, heparanase; CRP, C-reactive protein; IL-6, interleukin-6. 
diseases of MIS was T2DM. In recent years, with the changes of the lifestyle and the acceleration of the aging in China, the prevalence of diabetes has soared from $9.7 \%$ in 2010 , to $10.4 \%$ in 2013 and to 11.2 in 2017 , and the prevalence of diabetes in the elderly over the age of 60 is over $25 \%$ in $2017 .{ }^{10}$ Diabetes often develop a variety of clinical complications, including AS, NAFLD and obesity, which have severely affected the health of diabetes patients. These four metabolic diseases often aggregate or accompany with each other, and their common pathogenesis and pathological characteristics are all related to CLGI. Therefore, these four diseases can be treated in the same way. For example, metformin has been proven in clinical work to reduce blood sugar, weight and the risk of cardiovascular events, and also reduce the effects of fatty liver. Of course, these four diseases can also be prevented in the same way. For example, aerobic exercise has a positive preventive effect on the four components of MIS. ${ }^{1}$ The pathogenesis of MIS is yet not completely clear, so finding out the relevant factors of MIS pathogenesis and targeted treatment will have a positive effect on the prevention and treatment of MIS.

The pathogenesis of MIS was based on CLGI. These four metabolic diseases were all related to CLGI, particularly T2DM and obesity. It was worth mentioning that obesity might lead to T2DM through a series of inflammatory reactions. ${ }^{11}$ Moreover, CLGI played a crucial role in all the stages of AS formation, implying that antiinflammatory therapy was of great significance for the treatment of atherosclerosis-related cardiovascular diseases. $^{12}$ Similarly, NAFLD was also associated with CLGI, and closely related to macrophage-mediated inflammation. ${ }^{13}$ Studies have demonstrated that adiponectin levels in MIS patients tend to be lower compared to those in non-MIS patients, indicating that adiponectin could be used as an indicator to predict the occurrence of MIS. ${ }^{14}$ The results of this study revealed that the serum HPA levels of MIS patients were obviously higher than those of non-MIS patients, hence serum HPA levels might also be used as a predictor of MIS occurrence.

HPA was an important functional enzyme in the human body, which has the function of specifically hydrolyzing HSPG. HPA was involved in the development and metastasis of many tumors, for example, it had shown an important role in the development and metastasis of gastric cancer and breast cancer. $^{15,16}$ At present, scholars had conducted a Phase II clinical trial of HPA inhibitor PI-88 for tumor treatment and obtained an ideal result. ${ }^{17}$ In addition, HPA was related to the development of tumors, also closely related to the development of inflammatory diseases. At present, a large number of related research reports have been reported. After degrading HSPG, HPA could participate in inflammatory responses from multiple aspects, such as leukocyte recruitment, spillage, and aggregation to the site of inflammation; it could also promote the release of cytokines and chemokines fixed on the extracellular matrix or cell surface, and activation of innate immune cells. ${ }^{18}$ HPA was involved in the occurrence and development of inflammatory diseases such as rheumatoid arthritis (RA) and inflammatory bowel disease (IBD). ${ }^{19,20}$ At present, studies have reported the correlation between HPA and inflammation-related diseases such as diabetes and AS. However, there was no report on the correlation between HPA and MIS, so this article explored the correlation between HPA and MIS and its possible mechanism.

The results of this study showed that serum HPA levels in the MIS group were significantly higher than those in the control group, indicating that higher HPA levels might play a certain role in the occurrence and development of MIS. Additionally, the expression levels of HPA in patients with metabolic syndrome remain unknown, which may require further research.

The nature of MIS was mainly CLGI mediated by macrophages. $^{21}$ C-reactive protein (CRP) was a plasma pentameric protein produced mainly by the liver. It was a very sensitive major protein involved in non-specific inflammatory or acute inflammatory response phases. Interleukin-6 (IL-6) was a cytokine with multiple functions and could be produced by cells such as monocytic macrophages, vascular endothelial cells, and was closely related to inflammatory responses. Studies have demonstrated that serum CRP and IL-6 levels rose significantly during CLGI and lasted for a long time, so CRP and IL-6 were considered to be two representative indicators of CLGI. $^{22}$ The serum CRP and IL-6 levels in patients with MIS would be detected to analyze the correlation between serum CRP and IL-6 levels and MIS, and we also would analyze whether there was an intrinsic link and correlation between serum CRP, IL-6 levels and serum HPA. For reason given above, serum CRP and IL-6 levels were also detected in this study. Serum CRP and IL-6 levels were two representative indicators of CLGI. The results of this study showed that serum CRP and IL-6 levels in the MIS group were significantly higher than those in the control group, further confirming that the essence of MIS 
was a CLGI. The Pearson correlation analysis showed that serum HPA levels were significantly positively correlated with CRP and IL-6 levels, indicating that there might be some internal upstream and downstream relationship between the expression of HPA and the secretion of inflammatory factors. HPA might be involved in the process of chronic low-grade inflammatory response in MIS.

Contrary to our expected results, the cholesterol and low density lipoprotein cholesterol of MIS groups were lower than that of the control group, which may be related to the long-term use of lipid-lowering drugs of MIS patients.

As a metabolic inflammatory disease, MIS might have potential therapeutic value through anti-inflammatory treatment. Metformin was a first-line drug for the treatment of T2DM and achieves hypoglycemic effects by increasing insulin sensitivity and reducing liver glucose output. Metformin could not only improve obesity symptoms and controlling metabolic inflammation, but also reduced the risk of cardiovascular disease. ${ }^{23,24}$ Therefore, metformin could have important therapeutic value for MIS by improving insulin resistance and reducing inflammation.

Aspirin was a traditional anti-inflammatory drug. Previous studies have shown that aspirin could reduce insulin resistance and improved glucose and lipid metabolism. ${ }^{25,26}$ Current research showed that aspirin could bind to the Glu225 region of HPA and inhibit HPA activity. ${ }^{27}$ Therefore, aspirin was expected to become a potential drug for the treatment of MIS. In addition, there were inhibitors of HPA PI-88, PG545, etc. At present, such HPA inhibitors have been studied more in the occurrence and development of tumors, and have been proven to inhibit tumor growth and metastasis. However, its research on inflammatory response was less. Therefore, the role of HPA inhibitors in inflammatory response needed to be further studied at present, and it might become a candidate drug for the treatment of MIS in the future.

In summary, the results obtained in this study revealed that compared with the control group, the serum HPA level was considerably higher in the MIS group, and the serum HPA level was significantly positively correlated with the CRP and IL-6 levels. These results suggest that higher serum HPA levels plays a certain role in the pathogenesis and development of MIS, and that serum HPA levels might interact with CRP and IL-6 or have an upstream and downstream regulatory relationship, and might participate in the CLGI process induced by MIS. This study provides a certain evidence and reference basis for further in-depth and systematic research on the CLGI effects of HPA in the future.

\section{Data Sharing Statement}

The data used to support the findings of this study are available from the corresponding author Houguang Zhou upon request.

\section{Ethics Declarations}

This study was performed in accordance with the Declaration of Helsinki (2013) of the World Medical Association, and approved by the ethics committee of Huashan Hospital, Fudan University (No.2015-127).

\section{Acknowledgments}

Ying-hong Tao and Hou-guang Zhou are co-corresponding authors. Qing-song Wang, Xiu-ling Zhou and Guang-hai Mei are co-first authors. This work was supported by grants from the National Natural Scientific Foundation, China (81871098, 81571361 and 81170322), Projects of Shanghai Health and Health Committee on Integration of traditional Chinese and Western Medicine (ZY(20182020)-FWTX-3007, ZHYY-ZXYJHZX-201915).

Shanghai Medical Park New Star-Outstanding Young Medical Talents Program, and Shanghai Municipal Key Clinical Specialty (Geriatrics, No. shslczdzk02802).

The authors would like to thank Dr Shouling Mi for general supervision of the study and insightful comments and detailed revisions to the manuscript.

\section{Disclosure}

The authors declare no conflicts of interest.

\section{References}

1. Hu RM. Concept and clinical significance of metabolic inflammatory syndrome. Clinical Focus. 2016;31(09):960-963.

2. Wang F, Wan A, Rodrigues B. The function of heparanase in diabetes and its complications. Can J Diabetes. 2013;37(5):332-338. doi:10.1016/j.jcjd.2013.05.008

3. Irving-Rodgers HF, Ziolkowski AF, Parish CR, et al. Molecular composition of the peri-islet basement membrane in NOD mice: a barrier against destructive insulitis. Diabetologia. 2008;51(9):1680-1688. doi:10.1007/s00125-008-1085-x

4. Shafat I, Ilan N, Zoabi S, Vlodavsky I, Nakhoul F. Heparanase levels are elevated in the urine and plasma of type 2 diabetes patients and associate with blood glucose levels. PLoS One. 2011;6(2):e17312. doi:10.1371/journal.pone. 0017312

5. Baker AB, Chatzizisis YS, Beigel R, et al. Regulation of heparanase expression in coronary artery disease in diabetic, hyperlipidemic swine. Atherosclerosis. 2010;213(2):436-442. doi:10.1016/j. atherosclerosis.2010.09.003 
6. Muhammad RS, Abu-Saleh N, Kinaneh S, et al. Heparanase inhibition attenuates atherosclerosis progression and liver steatosis in E0 mice. Atherosclerosis. 2018;276:155-162. doi:10.1016/j. atherosclerosis.2018.07.026

7. Colman PG, Thomas DW, Zimmet PZ, Welborn TA, Garcia-Webb P, Moore MP. New classification and criteria for diagnosis of diabetes mellitus. The Australasian Working Party on Diagnostic Criteria for Diabetes Mellitus. N Z Med J. 1999;112(1086):139-141.

8. Review T, LaBrecque DR, Abbas Z, et al. World Gastroenterology Organisation global guidelines: nonalcoholic fatty liver disease and nonalcoholic steatohepatitis. J Clin Gastroenterol. 2014;48 (6):467-473. doi:10.1097/MCG.0000000000000116

9. Kinoshita M, Yokote K, Arai H, et al. Japan Atherosclerosis Society (JAS) Guidelines for Prevention of Atherosclerotic Cardiovascular Diseases 2017. J Atheroscler Thromb. 2018;25(9):846-984. doi:10.5551/jat.GL2017

10. Li Y, Teng D, Shi X, et al. Prevalence of diabetes recorded in mainland China using 2018 diagnostic criteria from the American Diabetes Association: national cross sectional study. BMJ. 2020;369:m997. doi:10.1136/bmj.m997

11. Aamir K, Khan HU, Sethi G, Hossain MA, Arya A. Wnt signaling mediates TLR pathway and promote unrestrained adipogenesis and metaflammation: therapeutic targets for obesity and type 2 diabetes. Pharmacol Res. 2020;152:104602. doi:10.1016/j.phrs.2019.104602

12. Nasonov EL, Popkova TV. Atherosclerosis: perspectives of anti-inflammatory therapy. Terapevt Arkh. 2018;90(5):4-12.

13. Lefere S, Tacke F. Macrophages in obesity and non-alcoholic fatty liver disease: crosstalk with metabolism. JHEP Rep. 2019;1 (1):30-43. doi:10.1016/j.jhepr.2019.02.004

14. Ma L, Xu Y, Zhang Y, Ji T, Li Y. Lower Levels of Circulating Adiponectin in Elderly Patients with Metabolic Inflammatory Syndrome: a Cross-Sectional Study. Diabetes Metab Syndr Obes. 2020;13:591-596. doi:10.2147/DMSO.S242397

15. Liu LP, Sheng XP, Shuai TK, Zhao YX, Li B, Li YM. Helicobacter pylori promotes invasion and metastasis of gastric cancer by enhancing heparanase expression. World J Gastroenterol. 2018;24 (40):4565-4577. doi:10.3748/wjg.v24.i40.4565

16. Sun X, Zhang GL, Nian JY, et al. Elevated heparanase expression is associated with poor prognosis in breast cancer: a study based on systematic review and TCGA data. Oncotarget. 2017;8 (26):43521-43535. doi:10.18632/oncotarget.16575
17. Khasraw M, Pavlakis N, McCowatt S, et al. Multicentre phase I/II study of PI-88, a heparanase inhibitor in combination with docetaxel in patients with metastatic castrate-resistant prostate cancer. Ann Oncol. 2010;21(6):1302-1307. doi:10.1093/annonc/mdp524

18. Meirovitz A, Goldberg R, Binder A, Rubinstein AM, Hermano E, Elkin M. Heparanase in inflammation and inflammation-associated cancer. FEBS J. 2013;280(10):2307-2319. doi:10.1111/febs.12184

19. Digre A, Singh K, Abrink M, et al. Overexpression of heparanase enhances $\mathrm{T}$ lymphocyte activities and intensifies the inflammatory response in a model of murine rheumatoid arthritis. Sci Rep. 2017;7:46229. doi:10.1038/srep46229

20. Quaglio AE, Castilho AC, Di Stasi LC. Experimental evidence of heparanase, Hsp70 and NF-kappaB gene expression on the response of anti-inflammatory drugs in TNBS-induced colonic inflammation. Life Sci. 2015;141:179-187. doi:10.1016/j.1fs.2015.09.023

21. Li RM, Hu RM. Progress in the pathogenesis of metabolic inflammatory syndrome. Fudan Univ J Med Sci. 2017;44(03):380-384.

22. van Woudenbergh GJ, Theofylaktopoulou D, Kuijsten A, et al. Adapted dietary inflammatory index and its association with a summary score for low-grade inflammation and markers of glucose metabolism: the Cohort study on Diabetes and Atherosclerosis Maastricht (CODAM) and the Hoorn study. Am J Clin Nutr. 2013;98(6):1533-1542. doi:10.3945/ajen.112.056333

23. Zheng S, Liu E, Zhang Y, et al. Circulating zinc-alpha2-glycoprotein is reduced in women with polycystic ovary syndrome, but can be increased by exenatide or metformin treatment. Endocr J. 2019;66 (6):555-562. doi:10.1507/endocrj.EJ18-0153

24. Schutt KA. [Evidence-based reduction of cardiovascular risk in patients with diabetes]. Herz. 2020;45(2):118-121. doi:10.1007/ s00059-019-04876-0

25. Abdin AA, Baalash AA, Hamooda HE. Effects of rosiglitazone and aspirin on experimental model of induced type 2 diabetes in rats: focus on insulin resistance and inflammatory markers. J Diabetes Complicat. 2010;24(3):168-178. doi:10.1016/j.jdiacomp.2009.01.005

26. Hundal RS, Petersen KF, Mayerson AB, et al. Mechanism by which high-dose aspirin improves glucose metabolism in type 2 diabetes. J Clin Invest. 2002;109(10):1321-1326. doi:10.1172/JCI0214955

27. Dai XY, Yan J, Fu XH, et al. Aspirin Inhibits Cancer Metastasis and Angiogenesis via Targeting Heparanase. Clin Cancer Res. 2017;23 (20):6267-6278. doi:10.1158/1078-0432.CCR-17-0242

\section{Publish your work in this journal}

Diabetes, Metabolic Syndrome and Obesity: Targets and Therapy is an international, peer-reviewed open-access journal committed to the rapid publication of the latest laboratory and clinical findings in the fields of diabetes, metabolic syndrome and obesity research. Original research, review, case reports, hypothesis formation, expert opinion and commentaries are all considered for publication. The manuscript management system is completely online and includes a very quick and fair peer-review system, which is all easy to use. Visit http://www.dovepress.com/testimonials.php to read real quotes from published authors. 